\title{
Acknowledgement to Reviewers of Fractal and Fractional in 2017
}

Fractal and Fractional Editorial Office

MDPI AG, St. Alban-Anlage 66, 4052 Basel, Switzerland

Published: 30 January 2018

Peer review is an essential part in the publication process, ensuring that Fractal and Fractional maintains high quality standards for its published papers. In 2017, a total of 17 papers were published in the journal. Thanks to the cooperation of our reviewers, the median time to first decision was 14 days and the median time to publication was 24 days. The editors would like to express their sincere gratitude to the following reviewers for their time and dedication in 2017:

\author{
Abd-Elhameed, Waleed \\ Aguilar, José Francisco Gómez \\ Ahmad, Fayyaz \\ Atangana, Abdon \\ Bazhlekova, Emilia \\ Błasik, Marek \\ Bountis, Tassos \\ Carlini, Maurizio \\ Cattani, Carlo \\ Chen, Wen \\ Chen, Xianghong \\ Concezzi, Moreno \\ Dassios, Ioannis K. \\ Datsko, Bohdan \\ Duarte-Mermoud, Manuel A. \\ Dubbeldam, Johan \\ Fabrizio, Mauro \\ Ferras, Luís Jorge Lima \\ Galhano, Alexandra \\ Gallagher, John \\ Georgiou, Georgios \\ Ibrahim, Rabha W. \\ Ingber, Lester \\ Jorgensen, Palle \\ Kirane, Mokhtar \\ Kobayashi, Motoyoshi \\ Lane-Serff, Gregory \\ $\mathrm{Li}$, Ming \\ Li, Xuemin \\ Liu, Da-Yan \\ Luchko, Yuri
}

Moroni, Monica

Nie, Yufeng

Nieto, Juan J.

Ogata, Satoshi

Olsen, Lars Ole Ronnow

Ostoja-Starzewski, Martin

Otto, Andreas

Povstenko, Yuriy

Rassias, Michael (Michail) Th.

Reich, Simeon

Rodino, Luigi

Rodrigues, Maria Manuela Fernandes

Rodríguez, Francisco

Sandev, Trifce

Schwarz, Phil

Sequeira, Adélia

Simsek, Yilmaz

Sivasundaram, Seenith

Spigler, Renato

Sumelka, Wojciech

Tarasov, Vasily

Ushida, Akiomi

Vieru, Dumitru

Villecco, Francesco

Vives, Josep

Wacławczyk, Marta

Wang, Yuanqi

Wolf, Marek

$\mathrm{Wu}$, Guo-Cheng

Yamano, Takuya

Yang, Xiao-Jun 
Machado, Tenreiro

Zorica, Dusan

Mainardi, Francesco

(C) 2018 by the authors. Licensee MDPI, Basel, Switzerland. This article is an open access article distributed under the terms and conditions of the Creative Commons Attribution (CC BY) license (http://creativecommons.org/licenses/by/4.0/). 